\title{
Mobile Agent Programming Paradigm and its Application Scenarios
}

\author{
Rahul Singh Chowhan* \\ SRF, Computer Science, Agriculture University, Jodhpur, India \\ *Corresponding author
}

\begin{tabular}{|l|}
\hline Key w or d s \\
Mobile Agent, \\
Programming \\
Paradigm
\end{tabular}

\author{
A B S T R A C T
}

Mobile agent technology offers another processing worldview in which a program, as a motoring tool or moving administrator, can suspend its execution on a host PC, exchange itself to another operator empowered host on the system, and resume execution on the new host. The utilization of portable code has a long history going back to the utilization of remote occupation passage frameworks in the 1960's. Web applications confront challenges that distributed protocols and the selection of improved coordination models may overcome the conventional challenges. More and more user level applications get involved into Internet from intranets and meanwhile the applications running over the Internet turn out to be more distributed and portable in nature of its execution. These new plans and programming models call for help to outfit the Web's potential for longer survival in competitive market. Technologies like Mobile Agent are becoming widely accepted by researchers and commercial brokers, as they are capable of performing well in client-server traditional framework as well as can be appropriated with an arrangement of procedures to a given execution condition by moving on to next neighbor and utilizing its assets. Interestingly, the portable agents characterizes applications as comprising of typical system elements which can show code versatility by concurrently changing their execution condition and exchanging themselves amid execution. The present agent oriented incarnations can be portrayed in various routes going from basic conveyed items to profoundly sorted out programming with implanted knowledge. As the modernity of agent oriented programming has expanded overtime. The article examines framework level issues and necessities for programming agents that emerge in the plan of general and specialized frameworks.

\section{Introduction}

Mobile agents can be referred to as programs that can maneuver through a network by themselves.

They are able to migrate from host to host and can interact with other resources and agents on each network. It is argued that these autonomous agents are able to provide an efficient, convenient and robust paradigm for programming of distributed applications. This is the case when there are partially connected computers involved. The partially connected computers may include modem connected computers, personal digital assistants and mobile computers such as laptops. These are often disconnected from the network. In this article we shall look at the properties and some of the benefits of mobile agent systems.

\section{Properties of agents}

Some of the key agent's attributes are; autonomous; this means that they are capable of autonomous actions that allow 
achievements of goals without the need of external interventions (Persson, 2000). They are also interactive; meaning they can communicate with the environment and other agents. They are also adaptive; meaning they can change and improve behavior by responding to changes. Other attributes include Intelligence; meaning they are capable of performing flexible and autonomous actions so as to achieve goals and this state is dictated by knowledge. They are also flexible; meaning they combine reactive, proactive and social attributes. They are also reactive; meaning they can monitor the environment and afterwards responding, replanning and reorganizing with the aim of adapting to the environmental changes (Persson, 2000). They are also proactive; meaning they set a plan so as to achieve a goal and they work towards the plan. They are social; meaning they can develop social relations. They are mobile; meaning they are capable of transporting themselves. They are trustworthy, meaning they are truthful and can be trusted because they don't act in unexpected manner.

\section{Object vs Agent}

The major difference between an agent and an object is that an agent resembles a human being and is often pursuing its own agendas while the object reflects the ideas of devices with the well-known interfaces and therefore it's an analogy to the real world object (Juziuk, 2012). One of the fundamental property of an object is that it is able to encapsulate some state, this means that the object has control over its own state, this is because there are some variables internally in the object and can only be accessed by that object. The agents also have their private state that is expressed by their beliefs. The objects also communicate by passing message that is represented, for example, method invocations (Juziuk, 2012). This is not the case with agents, which are more flexible and they do not act on every request but instead first decide whether it is necessary.

\section{Benefits of using mobile agents}

The technology of mobile agents can be exciting but our interest in the mobile agents should not be in any way motivated by this technology, but rather it should be because of the benefits that they provide by creation of distributed systems. These are some of the good reasons why you should be using mobile agents (Chennamaneni, 2011).

They help reduce network load: the distributed systems will often rely on the communication's protocols that will involve multiple interactions so as to accomplish a task. This is often applicable when the security measures have been enabled. This results to a lot of traffic to the network. What mobile agents do is that they allow you be able to package a conversation and then dispatch it to the destination host where the interactions are able to take place locally. The mobile agents are also important when reducing the flow of raw data in a network (Chennamaneni, 2011). When very large volumes of data are stored in the remote hosts, then these data should be processes within the locality of the data instead of transferring to the network, the motto followed is simple, you should move the computations to the data and not the data to the computations.

\section{Mobile agents overcome network latency}

Some of the critical real time systems, such as robots involved in the manufacturing process require to respond to the changes in their environments in real time. The controlling of such a system through the factory network of a substantial size involves significant latencies. What the mobile agents do is that 
they offer a solution, the reason being that they can be dispatched from the central controller to directly execute the controllers' directions and act locally (Chennamaneni, 2011).

\section{Mobile agents encapsulate protocols}

When there is an exchange of data in a distributed system, each of the hosts owns the code that then implements the required protocols for coding the outgoing data and also interpreting the incoming data respectively. On the other hand as protocols evolve so as to accommodate security or efficiency requirements, it becomes difficult if not an impossible task to upgrade the protocol code the right way. This results to the protocols becoming a legacy problem (Lange, 2014). On the other hand mobile agents are capable of moving remote hosts so as to establish the required channels that are based on proprietary protocols.

\section{Mobile agents execute autonomously and asynchronously}

Most of the times mobile devices have to rely on fragile and expensive network connections. For instance, a task that requires a continuous open connection between a fixed network and a mobile device will often not be technically or economically feasible.

This means that tasks can be emended into the mobile agents which afterwards can be dispatched into the network. After dispatch, the mobile agents then become independent of the process of creating and can therefore operate autonomously and asynchronously (Lange, 2014).

\section{Mobile agents adapt dynamically}

They have the capability of sensing their environment of execution and therefore react autonomously to any changes (Lange, 2014). Multiple mobile agents have the ability to distribute themselves within the hosts in the network in a way that allows the maintaining of the optimal configurations responsible for solving any particular problem.

\section{Mobile agents are naturally heterogeneous}

From both the hardware and software perspective, network computing is basically heterogeneous (Lange, 2014). The mobile agents are generally computer and transport layer independent and are only dependent on the environment that execution takes place, they therefore provide the optimal conditions that allows for the integration of a system seamlessly.

\section{Mobile agents are fault tolerant and robust}

Mobile agents have the ability to react dynamically to unfavorable events and situations and therefore making it easier to build distributed systems that are robust and fault tolerant. For instance, when a host is in the process of being shut down, all the agents that are executing on the machine are warned and allowed time for dispatching and continuing their operations on the other hosts within the network (Chennamaneni, 2011).

\section{Mobile agents adaptation and navigation}

Uncertainty and the need for being dynamic is common in the world of mobile agents. This is because machines move up and down as the data stored in the repositories is changed. The exact sequence of destinations and the required steps so as to complete any information gathering task is most of the time not completely known during the launch of the agent into the world. Therefore, an autonomous agent can be crippled without external state, this is because it has no way of perceiving and then adapting to the dynamic 
changes in the environment (Lange, 2014). There are some sensors that help an agent in determining its external state and there are mechanisms that use these sensors for the purpose of adaptive navigation.

\section{Network sensing}

This is the ability of a mobile device to detect the state of its network connection. It has a more important function, but when it comes to provision of information to the agent about its expected transit time across the network and about as to whether the network site is reachable by any means (Lange, 2014). This kind of information enables agents to adapt to any changes in the network conditions. Let's consider an agent that requires to visit information resources and several sites. What a smart agent will do is that it will be able to adapt to the fact that some of the sites may currently be unreachable and therefore will visit other sites first. A smarter agent on the other hand will have the ability to plan a sequence of visits with an estimation of each site's current network delay. Some of the other agents may opt to tailor their behavior according to the current bandwidth that is available, this may be the amount of data they carry along. Some of the tools include; 1) a tool that enables the determining as to whether the local host is connected (Lange, 2014). It pings the broadcast address on the local subnet; if the response is within a short time, it connects the network. 2) A tool that detects as to whether a specific host is reachable, it is the standard ping. 3) A tool that determines the expected bandwidth to a remote host, therefore allowing hosts to choose their destination or the amount of data based on bandwidth (Lange). Instead of measuring the bandwidth by sending lots of data to a remote host which will often take the same amount of time as sending directly to the agent, the bandwidth is predicted by experience.

\section{Application of mobile agents: a scenario for an airline ticketing system}

When a user intends to purchase an airline ticket through the internet, he is provided with an array of options so as to plan his travel in the most efficient way. In the current modern world, an airline ticket should not be mistaken as only a booklet transporting a user from one place to the other, instead it is considered a lifestyle guide (Persson, 2000). If you look at any airline ticketing website, for instance, www.orbitz.com, it allows the traveler to have access to several options of hotel room bookings, car hire options, sightseeing tours, cruise bookings along with all their tickets. It's common for travel agencies to compete against each other by offering travel packages so as to expand their customer base and their business turnover (Persson, 2000). This may be in form of discounts awarded to the travelers that encourages them to extend their tours with an added holiday at a lesser cost. These kind of offers have made the online airline ticketing industry very competitive and complicated. This article will look at different considerations that influence the selling of airline tickets using an online based system. These considerations are viewed from both the airline and the customer perspective.

\section{The perspective of the customer}

Because airline travel is an expensive proposition, the customer seeks to get the best value for their money and therefore will evaluate various choices before deciding on which particular airline or service to use. For instance, let's consider Sam who flies frequently and also uses the web regularly, instead of using a travel agent who will charge him a huge commission, he uses the web to determine the best travel deals (Juziuk, 2012). When he is making a travel booking, Sam's key considerations are as follows; 1) the travel itinerary conforms to his travel 
needs. 2) When compared to the other rates, the cost of the ticket should be reasonable. 3) The airline company should have a reliable past record 4) his special preferences should be met by the airline service.

\section{The perspective according of the airline}

The airline perspective is mostly capital driven and therefore will have a few more aspects than that of the customer (Zaslavsky and Indrawan, 2016). To enable customers to access their services the airlines will allow the prospective traveler to choose from a few travel options. Most of these airlines understand that online ticketing is now common and the cheapest method of booking an airline ticket. The challenge that they face is how they would reach the implementation scenario. The agent system is responsible for creating a community of agents. The basic goal of the community is to maximize the agent's business, for instance, ticket booking agents, information searching agents, advertising agents, query handling agents which all form their own communities. These sub communities will then have community specific goals which when it comes to the super community will be ranked as secondary goals (Zaslavsky and Indrawan, 2016). For example, the advertising agents responsible of advertising the current services and schemes being offered will require a list of addresses so as to post their advertisement. An auxiliary agent can provide this list to the community agent responsible for advertising. In a similar manner, many interactions exists and occur within an agent space that contribute towards the successful realization of a super community goal. To sum up, this article has focused on defining what mobile agents are, we have seen that they are agents that are not bound to the system where execution begins. They have the unique ability to transport themselves from one system of the network to the other. We have also given some of the benefits of using a mobile agent, which include; reducing network load, overcoming network latency, they encapsulate protocols, they execute autonomously and asynchronously, adapt dynamically, naturally heterogeneous, and finally they are robust and fault tolerant. We have also looked at the difference between an agent and an object. Also we have looked at mobile agents applications. Then finally we have examined two different perspectives of mobile agents' application, namely the customer and airline perspective. This article will give the reader a basic but not full understanding of the broad topic of mobile agents.

\section{References}

Chennamaneni, A. (n.d.). An Overview of Mobile Agents in Mobile Computing.

Lange, D. B. (n.d.). Mobile object and Mobile Agents; The Future of Distributed Computing? General Magic Inc.

Page, J., Zaslavsky, A., \& Indrawan, M. (n.d.). A Buddy Model of Security for Mobile Agent Communities Operating in Pervasive Scenarios. Monash University, Melbourne, Australia.

Persson, M. (2000, December). Mobile Agent Architectures. Defence Research Establishment.

Juziuk, J. (2012, June 26). Design Pattterns for Multi-Agent Systems. Linnaeus University.

\section{How to cite this article:}

Rahul Singh Chowhan. 2018. Mobile Agent Programming Paradigm and its Application Scenarios. Int.J.Curr.Microbiol.App.Sci. 7(05): 3269-3273.

doi: https://doi.org/10.20546/ijcmas.2018.705.382 